\title{
Fault-tolerant almost exact state
}

\section{transmission}

SUBJECT AREAS:

QUANTUM INFORMATION

QUBITS

OPTICS AND PHOTONICS

NANOSCIENCE AND TECHNOLOGY

Received

22 March 2013

Accepted

17 October 2013

Published

4 November 2013

Correspondence and requests for materials should be addressed to

L.-A.W. llianaowu@ gmail.com)

\author{
Zhao-Ming Wang ${ }^{1,2}$, Lian-Ao Wu ${ }^{2,3}$, Michele Modugno ${ }^{2,3}$, Wang Yao ${ }^{4} \&$ Bin Shao ${ }^{5}$
}

'Department of Physics, Ocean University of China, Qingdao, 266100, China, ${ }^{2}$ Department of Theoretical Physics and History of Science, University of the Basque Country UPV/EHU, 48008, Spain, ${ }^{3}$ IKERBASQUE, Basque Foundation for Science, 48011 Bilbao, Spain, ${ }^{4}$ Department of Physics, The University of Hong Kong, Hong Kong, China, ${ }^{5}$ Key Laboratory of Cluster Science of Ministry of Education, and Department of Physics, Beijing Institute of Technology, Beijing 100081, China.

We show that a category of one-dimensional $X Y$-type models may enable high-fidelity quantum state transmissions, regardless of details of coupling configurations. This observation leads to a fault-tolerant design of a state transmission setup. The setup is fault-tolerant, with specified thresholds, against engineering failures of coupling configurations, fabrication imperfections or defects, and even time-dependent noises. We propose an experimental implementation of the fault-tolerant scheme using hard-core bosons in one-dimensional optical lattices.

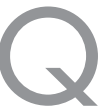
uantum devices require fault tolerant designs to tackle fabrication defects and environmental impacts, as exemplified by the fault-tolerant quantum computer. One of such quantum devices is a physical setup that reliably transmits a quantum state from one location to another. The quantum state transmission (QST) may be made through spin chains, or by ultracold atoms in one-dimensional (1D) optical lattices ${ }^{1-5}$. The quality of a QST depends on the coupling configurations, i.e., the coupling or tunnelling constants $J_{i, i+1}$ as a function of site indices $i$. It has been demonstrated that a QST cannot be achieved with perfect fidelity in a uniform spin chain with $X Y$ spin couplings, whereas it is possible in two cases where individual couplings are specially engineered: perfect state transfer (PST) ${ }^{6}$ and high-fidelity state transfer using weakly coupled external qubits ${ }^{7,8}$. The fidelity of the latter can be achieved with arbitrary precision. It has been widely believed that most configurations do not enable high-fidelity QSTs ${ }^{3,9}$.

This paper carefully examines diverse coupling configurations. To our surprise, the fidelity of QST is hardly determined by details but rather on the general architecture of $J_{i, i+1}$. Most configurations with larger values of $J_{i, i+1}$ in middle sites of the chains work equally well. In other words, having particular configurations, smaller on the ends and bigger in the middle, is crucial for enabling high-fidelity state transmission, regardless of specific details of the couplings. We find that the fidelity is fault-tolerant against random perturbations to coupling constants and site energies due to the fabrication processes. We also take into account the time-dependent random noise, which simulates the noisy effects due to environmental variables. Significantly, we show that the quantum states transmissions are hardly influenced by time-dependent noise. We then specify the faulttolerant thresholds for these failures, defects and noises. This observation leads to a fault-tolerant design of state transmission setup, which is robust against engineering failures in the coupling configurations, fabrication imperfections or defects, and even time-dependent noise. Unlike the strategy in fault-tolerant quantum computer, our proposal does not require dynamical control and relies only on natures of the setup. It is a self-protected quantum device.

We suggest that this setup could be realised experimentally by means of ultracold bosons in $1 \mathrm{D}$ optical lattices, as in the hard-core limit this system can be mapped into an effective spin one-half $X Y$ model $^{12}$. This system could be engineered by using the standard optical lattice technology and additional laser beams for tuning the couplings $J_{i, i+1}$ individually. It might be more feasible to use the spatial light modulator technology, that in principle allows to create arbitrary potentials and couplings for ultracold atoms ${ }^{17-19}$, and could be used to design specific coupling configurations. The fault tolerance ensures high-fidelity QST in presence of engineering failures, fabrication imperfections or defects, and noise.

Consider a spin chain described by a ferromagnetic Hamiltonian with couplings $J_{i, i+1}$ between sites $i$ and $i+1$,

$$
H=-\sum_{i=1}^{N-1} J_{i, i+1}\left(X_{i} X_{i+1}+Y_{i} Y_{i+1}\right) \text {, }
$$


where $J_{i, i+1}$ are allowed to be arbitrary. The conventional $X Y$ model is a special case when $J_{i, i+1}=$ constant. $X_{i}, Y_{i}$ are the Pauli matrices acting on spin $i$. $N$ is the size of the spin chain. We use $N=130$ for numerical illustrations, except examples where we show that the results presented here are almost size-independent. The $z$-component of the total spin or magnon is conserved. The model is the hardcore boson limit of the Bose-Hubbard Hamiltonian, as discussed later.

In quantum information theory, quantum state transfer often refers to transferring an unknown state, which is written as $|\phi(0)\rangle$ $=\alpha|0\rangle+\beta|1\rangle$ and is initially encoded in the first spin of the chain. Here $|0\rangle$ and $|1\rangle$ correspond to spin down and up states, respectively. The state $|\phi(0)\rangle$ is transferred to the other end as a result of free spin evolution. The fidelity of quantum state transfer is $f=\left.|| \alpha\right|^{2}+F e^{i \delta}$ $|\beta|^{2} \mid$, where $F$ is the fidelity of transmitting a known state $|1\rangle$ from the first spin to the last. The additional phase $\delta$ could be removed by applying a phase gate $\exp (-i \delta)$ to the last site as in ref. 6 . When $F=$ 1 , the transmission is exact ${ }^{4}, f=1$. Normally, $F$ is considerably smaller than $f$. For instance, an almost perfect state transfer $f=$ 0.97 for the state with $|\beta|^{2}=1 / 3$ only requires $F=0.9$. We will therefore focus on almost exact state transmissions with maximal fidelity $F_{\max }>0.9$ (see ref. 20), where $f$ could be more than 0.97 . In practice, most values of $F_{\text {max }}$ considered in the present paper are greater than 0.95 .

The Hamiltonian can be numerically diagonalized, specifically $H$ $=W H_{d} W^{\dagger}$, where $H_{d}$ is diagonal. The propagator of the Hamiltonian is therefore $U(t)=e^{-i H t}=W e^{-i H_{d} t} W^{\dagger}$. With this propagator, we can simulate the time evolution of quantum states for various coupling configurations.

\section{Results}

Fault-tolerant QST: configurations. We start by considering the following configuration of the couplings in eq. (1), $J^{w}(i) \equiv\left\{J_{1,2}=\right.$ $J_{N-1, N}=J_{0}, J_{i, i+1}=J$ elsewhere $\}$, that will be referred to as the weakcoupling limit. For simplicity, we fix $J=\hbar=1$ throughout this paper, without loss of generality. This corresponds to expressing time in units of $\hbar / J$. In Fig. 1 we show a density plot of the fidelity as a function of time and of the ratio $J_{0} / J$. This figure shows that, for fixed values of $J_{0} / J$ the fidelity reaches a maximal value $F_{\max }$ at specific times. The regions corresponding to an almost exact state transmissions $\left(F_{\max }>0.9\right.$ ), are coloured in red. With increasing $J_{0} / J$, $F_{\max }$ decreases, indicating that weaker couplings at the two ends are in favour of an almost exact state transmission (e.g. $F_{\max }=0.95$ at $J_{0} / J$ $=0.05$ ). In particular, we find that the maximal value for having $F_{\max }$

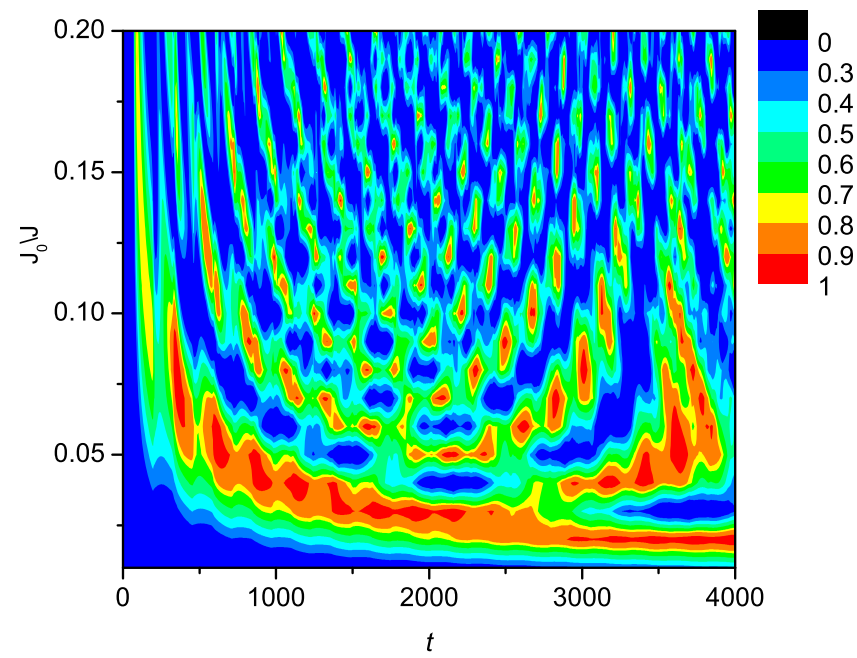

Figure $1 \mid$ Density plot of the fidelity as a function of time $\boldsymbol{t}$ and $J_{0} / J$. Here and in all figures time is expressed in units of $\hbar / J, J_{1,2}=J_{N-1, N}=J_{0}$.
$>0.9$ is $J_{0} / J=3 / 20$. Our numerical findings are consistent with the results of the perturbation theory in ref. 8 . In addition, the time $t_{\mathrm{MF}}$ at which the first maximal fidelity appears, decreases with $J_{0} / J$ as $t_{\mathrm{MF}} \propto$ $J / J_{0}$.

We now turn to other coupling configurations in the regions of the two ends, shown in Fig. 2(a). Configurations $\{1\},\{2\}$ and $\{4\}$ possess inversion symmetry with respect to the centre of the chain, and are all characterised by a similar efficiency in the state transmission. This is shown by Fig. 2(b), where we plot the time evolution of the fidelity (here for $J_{0} / J=0.05$ ). In all three cases, though the oscillation patterns are dramatically different, the fidelity reaches a maximal value $F_{\text {max }} \approx 0.95$. Instead, in the case of configuration $\{3\}-$ that is not invariant under parity - the maximal fidelity is low, $F_{\max }=0.28$.

From the experimental point of view, it may be easier to implement QSTs if sender's and receiver's sites are not at the ends but inside the chain. In order to analyse this case, we consider two further configurations, with the sender at the fourth site and the receiver at site $N-3$ (with different coupling arrangements), cases $\{5\}$ and $\{6\}$. The time evolution of the corresponding fidelity is shown in Fig. 2(c). This figure suggests that when the sender and receiver are weakly coupled to both their nearest neighbours, an almost exact state transmission can be achieved $\left(F_{\max }=0.95\right.$ in the configuration

$\{6\}$
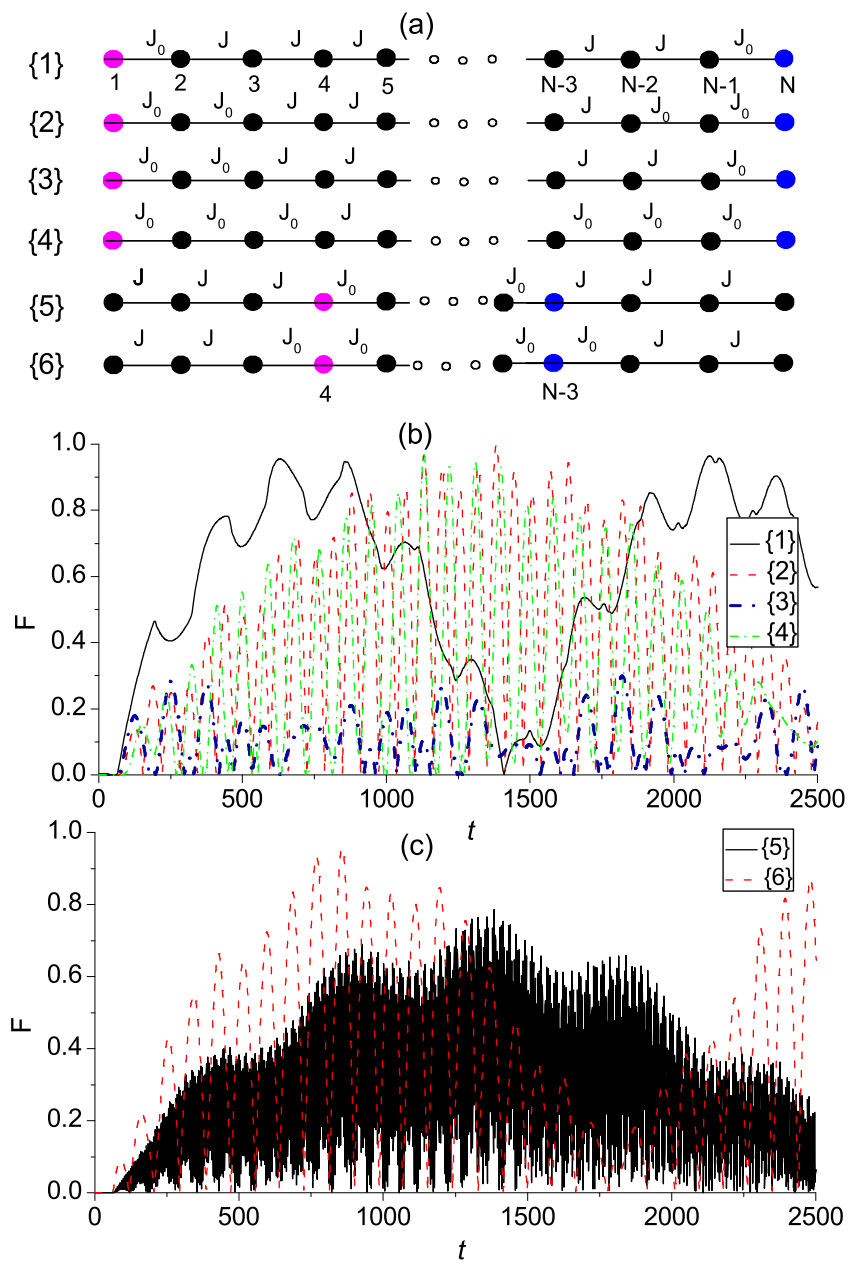

Figure $2 \mid$ (a) Different configurations of the couplings $J_{i, i+1}$. Magenta and blue colours are used to differentiate the sender's and the receiver's sites. (b) The fidelity as a function of time $t$ for cases $\{1-4\}$, for $J_{0} / J=0.05$. (c) The fidelity as a function of time $t$ for cases $\{5\}$ and $\{6\}$, with the same parameters as in (b). Note that in case $\{5\}$ there is only one neighbour weak coupling $J_{0}$ at the sender's or the receiver's sites while there is two in case $\{6\}$. 

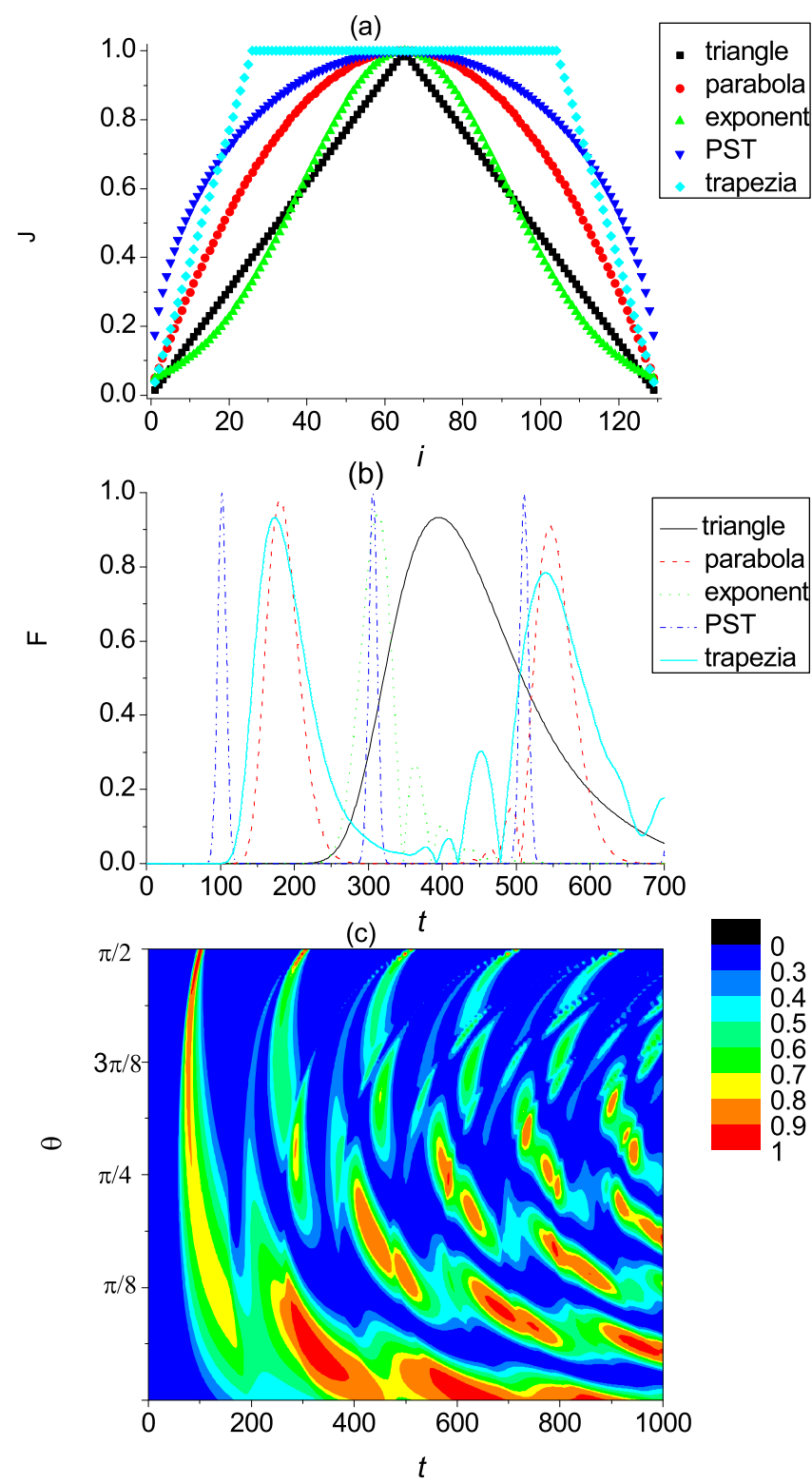

Figure $3 \mid$ (a) Coupling functions $J_{i, i+1}$. (b) Corresponding fidelities as a function of time $t$ for different $J_{i, i+1}$. (c) The contour fidelity as a function of time $t$ and $\theta$ when the coupling constants are given by $J_{i, i+1}=2 \sin \theta J \sqrt{i(N-i)} / N+\cos \theta J^{w}(i)$, where $\theta$ is a parameter for the interpolation.

$\{6\}$ ). Instead, when the outer coupling are stronger (case $\{5\}$ ), the transmission inside the chain - between sender and receiver - is weakened. This is verified by our numerical calculations.

Fault-tolerant QST: different types of $J$. We now consider different configurations of the couplings $J_{i, i+1}$, see Fig. 3(a). Namely, we analyse the cases of a triangle configuration $J_{i, i+1}=(2 J / N) \min (i, N$ $-i)$, parabola $J_{i, i+1}=-0.95 J(i-N / 2)^{2} /(1-N / 2)^{2}+J$, exponential $J_{i, i+1}=J \exp \left[(\ln 0.05)(i-N / 2)^{2} /\left(\begin{array}{lll}1 & \left.-N / 2)^{2}\right], \quad \text { PST }\end{array}\right.\right.$ $J_{i, i+1}=2 \sqrt{(i(N-i))} / N$, and trapezia $J_{i, i+1}=\{1$ for $i \in[26, \ldots$, $104], \min (i, N-i) / 26$ otherwise $\}$. These functions are normalised so that they have the same maximal values. The time evolution of the corresponding fidelities is plotted in Fig. 3(b). This figure shows that all these configurations yield excellent fidelities, the less efficient case being the triangle, with $F_{\max } \approx 0.94$. It is clear that an analytical interpolation between any two of these functions will work equally

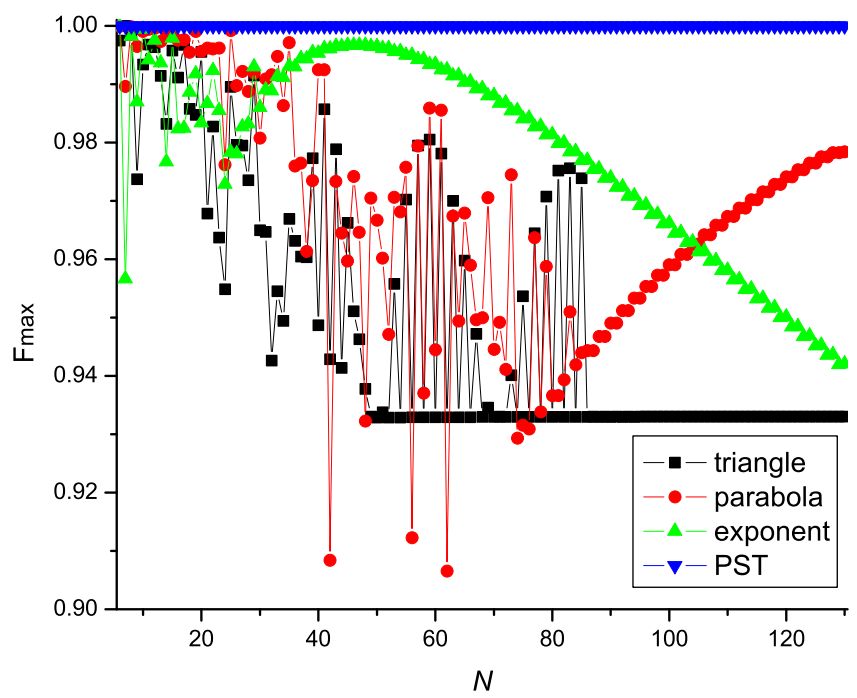

Figure $4 \mid$ Maximal fidelity $F_{\max }$ as a function of the chain length $N$, for different coupling configurations.

well, as exemplified in the following. Both the weak coupling limit, $J^{w}(i)$, and the PST configurations lead to excellent state transmissions with $F_{\max } \approx 1$. A possible interpolations between the two limits is given by $J_{i, i+1}=2 \sin \theta J \sqrt{i(N-i)} / N+J^{w}(i) \cos \theta$, with $\theta \in[0$, $\pi / 2$ ]. A density plot of the corresponding fidelity as a function of time and of the interpolation parameter $\theta$ is shown in Fig. 3(c). This figures shows that, though $t_{\mathrm{MF}}$ has a non trivial dependence on $\theta$, the maximal fidelity remains high for all values of $\theta$, as expected.

Significantly, these results are almost size-independent. This can be inferred from Fig. 4 where we plot the maximal fidelity $F_{\text {max }}$ versus $N$, for different coupling configurations (extracted from a time window $[0,4000])$ : in all cases $F_{\max }$ is well beyond $90 \%$.

Fault-tolerant QST against fabrication defects and dynamic noises. Random defects are unavoidable in the fabrication process. Therefore, it is crucial to have fault-tolerant mechanism for QSTs to address these imperfections ${ }^{10}$. Here we start by considering static random imperfections of both the tunnelling and the onsite energies, that are modelled as $J+\eta \operatorname{rand}(i)$ and $H_{\text {site }}=\epsilon \sum{ }_{i} \operatorname{rand}(i) Z_{i}$, respectively. Here rand() is a random function uniformly distributed in $[-1,1], \eta$ and $\epsilon$ are the corresponding amplitudes, and $Z_{i}$ is the Pauli matrix at position $i$. Numerically, we find that for $\eta, \epsilon<J / 20$ there is almost exact state transmission $\left(F_{\max }>0.9\right)$ for both the weak coupling and PST configurations, as well as for the interpolations discussed above.

Another important source of disturbance of our QST setup may be due to random noises from the environment, causing time-dependent fluctuations of the tunnelling coefficients and of the on-site energies. These effects can be easily included in our numerical scheme, see Methods. The results are shown in Figs. 5 and 6, where we draw a density plot of the fidelity as a function of time and of the fluctuation amplitudes (for the latter we use the same symbols $\eta$ and $\epsilon$ as in the static case), for $N=20$ and $N=130$ respectively. These figures show that in the weak coupling limit (panels (a) and (b)), a high-fidelity QSTs occurs up to $\eta, \epsilon \approx 0.05 \mathrm{~J}$, and that the noise in the on-site energies is somehow more effective in ruining QST. Instead, the PST configuration is much more robust against noise. In addition, by comparing the two figures, it is evident that the state transmission with shorter chain $N=20$ is slightly better than that with the long chain $N=130$ because of longer exposure to noise, in particular for the PST configuration. The quality of the weak coupling QST are 
(a)

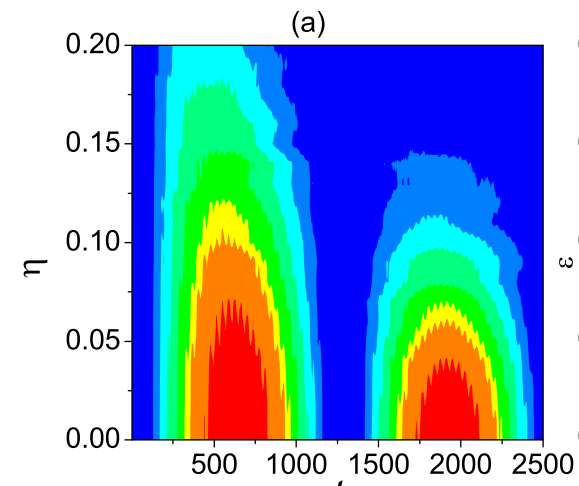

(c) $t$

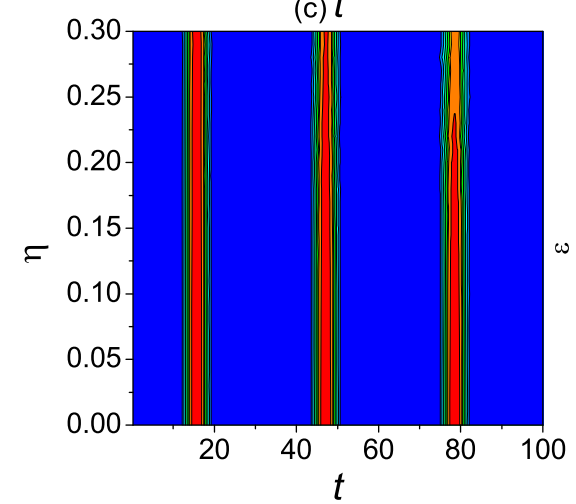

(b)

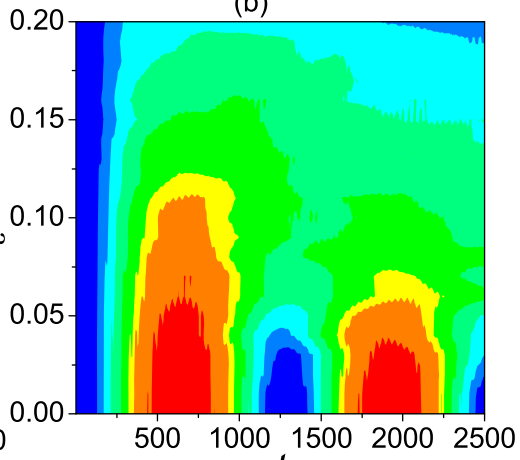

(d) $t$

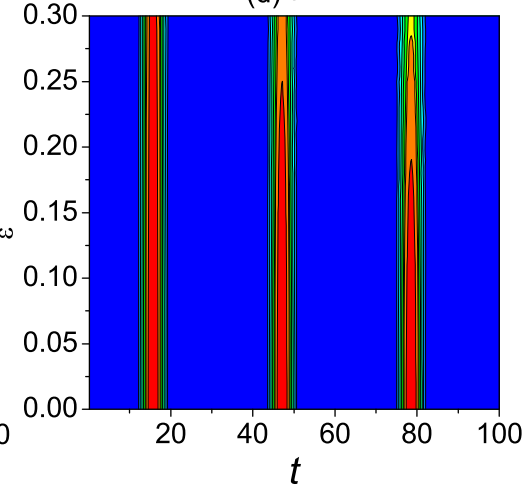

Figure $\mathbf{5}$ Fidelity vs. time $\boldsymbol{t}$, for time-dependent noise and with $\mathbf{N}=\mathbf{2 0}$. (a) Weak coupling limit with couplings perturbed by noise, (b) weak coupling limit with on-site noise, (c) PST with couplings perturbed by noise, (d) PST with on-site noise.
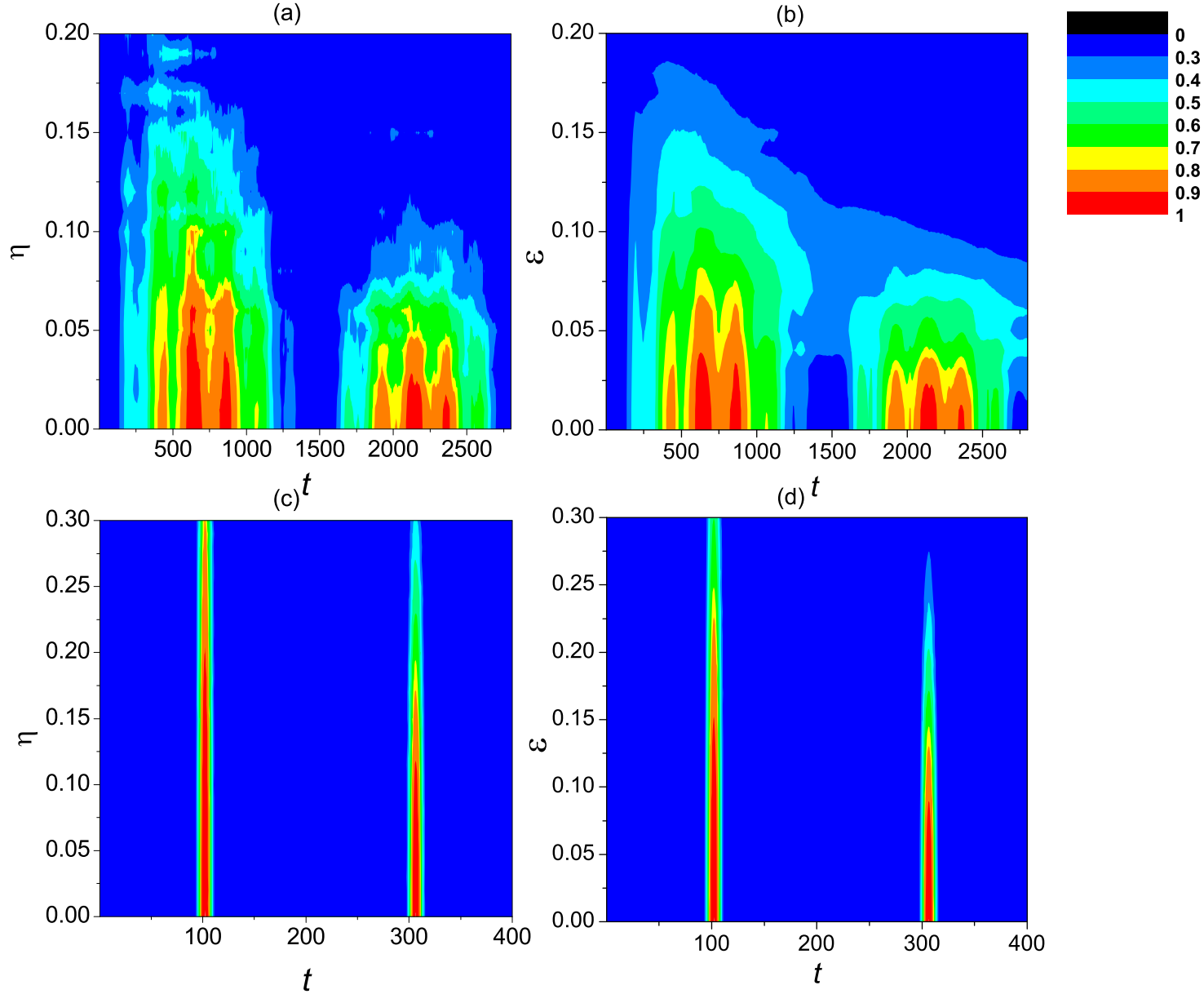

Figure $6 \mid$ The same as of Fig. 5, here for $N=130$. 
(a)

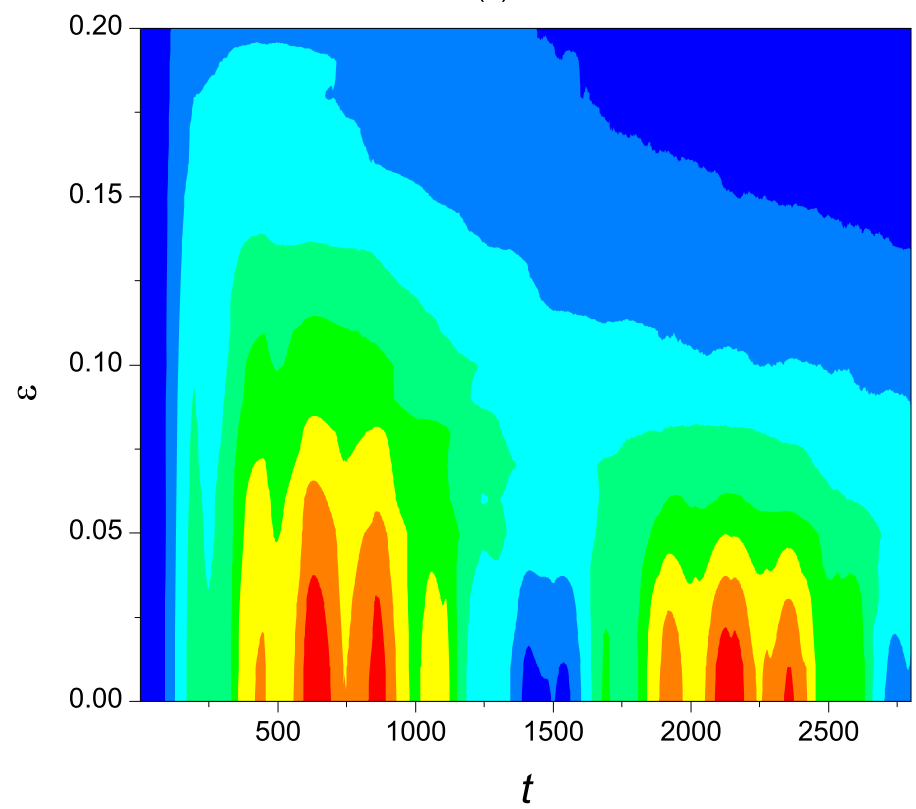

(b)

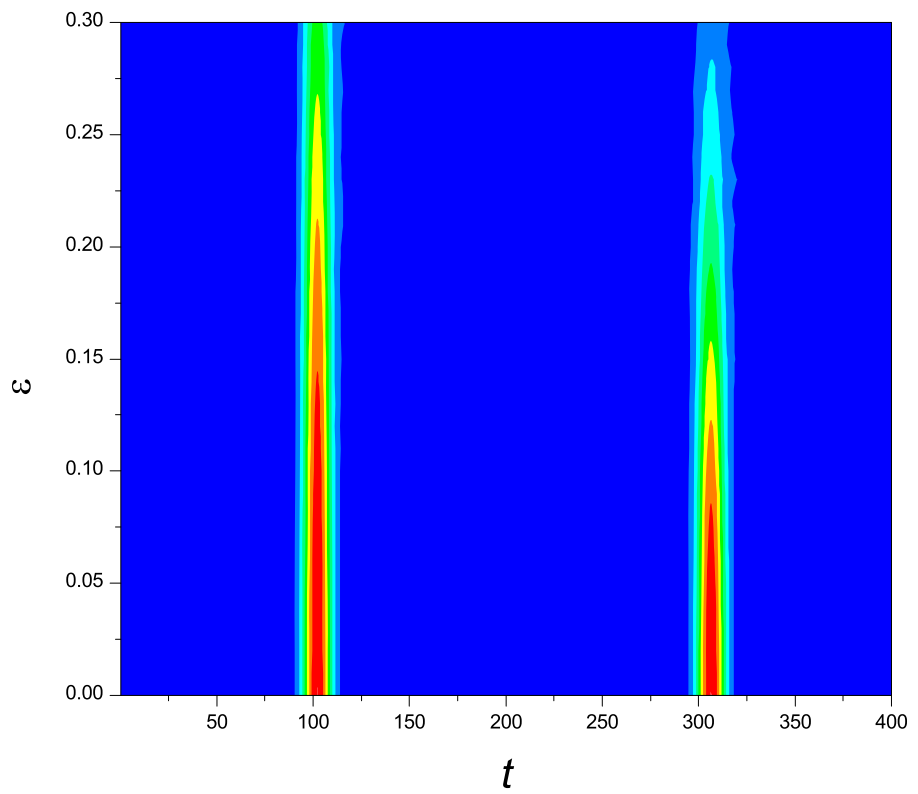

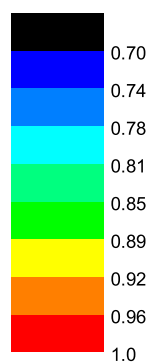

Figure 7 | Maximal average fidelity vs. time $\boldsymbol{t}$, for time-dependent on-site noise. (a) Weak coupling; (b) PST for $N=130$.

mostly determined by the ratio $J_{0} / J$ and the size effect is rather weak. Besides, peaks of the fidelity decreases with time as expected.

We remark that the time dependent on-site noises generate a phase difference between states $|0\rangle$ and $|1\rangle$. The acquired phases depend on time and on the initial state. Ref. 1 suggests an average fidelity to consider contributions from different initial states, which can be maximised by applying a proper magnetic field along $z$ direction. We calculate the maximal average fidelity over all possible input states in the presence of on-site noise, shown in Fig. 7. The overall picture is similar to the cases shown in the previous figures. We have checked that this would be the case for different noise realisations (see Methods).

To understand this fault-tolerance behaviour, we can break the QST into three stages: (1) the sender qubit emits its spin excitation into the spin chain in a timescale $\sim J_{0}^{-1} ;(2)$ the excitation propagates in the spin chain towards the receiver qubit with a propagation time determined by the spin-wave dispersion; (3) the receiver qubit absorbs the spin excitation. The second stage is affected by the time-independent random defects and time-dependent noise, but they both conserve the spin excitation number in the spin chain. Thus, the spin excitation emitted by the sender can still reach the receiver side in the presence of defects or noises as long as the disorders are not strong enough to change the spin excitation from extended modes to localised modes.

An experimental proposal. Let us now consider a specific experimental implementation by means of single-species ultracold bosons in a 1D tight-binding optical lattice ${ }^{11}$ (see also refs. 14, 15 for related proposals). An optical lattice can be created by using a retroreflected laser beam of wavelength $\lambda$, and is described by a potential $V(x)=s E_{R} \sin ^{2}(k x)$, with $k=2 \pi / \lambda$ and $E_{R}=\hbar^{2} k^{2} / 2 m$ being the the recoil energy. The bosons are also subjected to a 
transverse confinement providing the $1 \mathrm{D}$ geometry. In the tightbinding regime this system is described by the following Bose-Hubbard Hamiltonian

$$
\hat{H}_{H B}=-J \sum_{i=1}^{N-1}\left[\hat{a}_{i}^{\dagger} \hat{a}_{i+1}+\text { h.c. }\right]+\frac{U}{2} \sum_{i} \hat{n}_{i}\left(\hat{n}_{i}-1\right),
$$

with a uniform tunnelling rate, $J / E_{R}=(4 / \sqrt{\pi}) s^{0.75} \exp (-2.07 \sqrt{s})$ (see ref. 16). In the hard boson limit, $U \gg J$, one can consider states with at most one boson per site and use the presence/absence of a boson at a site to encode a quibit. The last term in (2) vanishes. In this regime the Hamiltonian can be cast in the form of the $X Y$ model in $(1)^{12}$. Next one may tune the couplings $J$ individually by focusing additional laser beams perpendicular to the lattice direction in correspondence of single sites. For sufficiently high intensities it is possible to create box-like barriers ${ }^{13}$. While this technique can create uniform couplings $J$ perfectly, the precise control of individual couplings, such as lattice ends or sites with a different coupling constant $J_{0}$, may be experimentally challenging. The major fault could happen when one uses the transverse lasers to address specific sites and locks these lasers in position, which may be tolerated in our scheme. On the other hand, a more promising technique is provided by the spatial light modulator (SLM) technology, that in principle allows to design arbitrary potentials for ultracold atoms ${ }^{17-19}$, and could be used to create one of our coupling configurations: smaller on the ends and bigger in the middle. This is a technique that is widely used for biological applications (see e.g. ${ }^{21}$ and references therein) and that is becoming to be available in experiments with ultra cold atoms. In addition, both techniques are subject to noises, e. g., from background gas, which could be tolerated in our design.

\section{Discussion}

We have demonstrated high-fidelity QST in an XY-type model, for a variety of coupling configurations. Besides the two excellent state transfer schemes - the PST and the weakly coupling limit - we have found that most symmetric configurations with larger values of the couplings $J_{i, i+1}$ inside the chain, present an almost exact state transmissions $\left(F_{\max }>0.9\right)$. We have found thresholds for enabling highfidelity QSTs with manufacturing imperfections and even in noise channels. We have also proposed a specific experimental implementation with hard-core boson in 1D optical lattices, designed by means of the current optical lattice technology or by using spatial light modulators.

\section{Methods}

Time-dependent noise. Two types of time-dependent noise are relevant for optical lattices: $i$ ) fluctuations of the lattice amplitude, affecting both the tunnelling and the on-site energies; ii) fluctuations of the position of the minima of the lattice. The latter cannot be accounted for in our approach, as the model is defined on a discrete lattice. Instead, we can address the effects of the former, by considering random fluctuations of both the couplings $J_{i, i+1}$ and the onsite energies. These are modelled as piece-wise for numerical convenience. Namely, the values of $J_{i, i+1}$ and of $H_{\text {site }}$ are considered constant over a short time interval $\tau$ (here we choose $\tau=0.1 \mathrm{~J} / \hbar$ ), and varied randomly between different time steps. From 0 to $\tau$, the evolution operator is $U(\tau)=\exp (-i H \tau)=W \exp \left(-i H_{d^{\tau}}\right) W^{\dagger}$. In the following time interval the Hamiltonian is changed to $H^{\prime}$ and the corresponding diagonal Hamiltonian is $H_{d}^{\prime}$, so that the evolution operator up to $2 \tau$ can be written $U(2 \tau)=W^{\prime} \exp \left(-i H_{d^{\tau}}^{\prime}\right) W^{\prime \dagger} W \exp \left(-i H_{d^{\tau}}\right) W^{\dagger}$. Further iterating the same procedure, one can evaluate $U(n \tau)$ for an arbitrary time step $n \tau$. Then, the density matrix of the whole system at time $t$ is evaluated by the ensemble average over different realisations of the noise (here we consider up to thousand noise realisations $)^{22}$. Eventually, we use the average density matrix to calculate the fidelity.
1. Bose, S. Quantum Communication through an Unmodulated Spin Chain. Phys. Rev. Lett. 91, 207901 (2003).

2. Wu, L.-A., Miranowicz, A., Wang, X. B., Liu, Y.-x. \& Nori, F. Perfect function transfer and interference effects in interacting boson lattices. Phys. Rev. A, 80, 012332 (2009).

3. Yung, H. Quantum speed limit for perfect state transfer in one dimension. Phys. Rev. A 74, 030303 (R) (2006).

4. Wu, L.-A., Liu, Y.-x. \& Nori, F. Universal existence of exact quantum state transmissions in interacting media. Phys. Rev. A 80, 042315 (2009).

5. Wang, Z.-M., Bishop, C. A., Byrd, M. S., Shao, B. \& Zou, J. Robust and reliable transfer of a qubit state through an XY spin chain. Phys. Rev. A 80, 022330 (2009).

6. Christandl, M., Datta, N., Ekert, A. \& Landahl, A. J. Perfect State Transfer in Quantum Spin Networks. Phys. Rev. Lett. 92, 187902 (2004).

7. Wojcik, A. et al. Unmodulated spin chains as universal quantum wires. Phys. Rev. A 72, 034303 (2005).

8. Oh, S. et al. Heisenberg spin bus as a robust transmission line for quantum-state transfer. Phys. Rev. A 84, 022330 (2011), and references therein.

9. Bayat, A., Banchi, L., Bose, S. \& Verrucchi, P. Initializing an unmodulated spin chain to operate as a high quality quantum data-bus. Phys. Rev. A 83, 062328 (2011).

10. Zwick, A., Álvarez, G. Z., Stolze, J. \& Osenda, O. Robustness of spin-coupling distributions for perfect quantum state transfer. Phys. Rev. A 84, 022311 (2011)

11. Bloch, I., Dalibard, J. \& Zwerger, W. Many-Body Physics with Ultracold Gases. Rev. Mod. Phys. 80, 885 (2008)

12. Lewenstein, M. et al. Ultracold atomic gases in optical lattices: mimicking condensed matter physics and beyond. Advances in Physics 56, 243 (2007).

13. Meyrath, T. P. et al. Bose-Einstein Condensate in a Box. Phys. Rev. A 71, 041604 (2005).

14. Banchi, L., Bayat, A., Verrucchi, P. \& Bose, S. Nonperturbative Entangling Gates between Distant Qubits Using Uniform Cold Atom Chains. Phys. Rev. Lett. 106, 140501 (2011).

15. Apollaro, T. J. G., Banchi, L., Cuccoli, A., Vaia, R. \& Verrucchi, P. 99\%-fidelity ballistic quantum-state transfer through long uniform channels. Phys. Rev. A 85, 052319 (2012).

16. Gerbier, F. et al. Interference pattern and visibility of a Mott insulator. Phys. Rev. A 72, 053606 (2005).

17. Boyer, V. et al. Dynamic Manipulation of Bose-Einstein Condensates with a Spatial Light Modulator. Phys. Rev. A 73, 031402(R) (2006).

18. Becker, C. et al. Oscillations and interactions of dark and dark-bright solitons in Bose-Einstein condensates. Nat. Phys. 4, 496 (2008).

19. Henderson, K., Ryu, C., MacCormick, C. \& Boshier, M. G. Experimental demonstration of painting arbitrary and dynamic potentials for Bose-Einstein condensates. New J. Phys. 11, 043030 (2009).

20. Liu, B.-Q., Wu, L.-A., Shao, B. \& Zou, J. General quantum-state swap: An X Ymodel analysis. Phys. Rev. A 85, 042328 (2012).

21. Grier, D. G. A revolution in optical manipulation. Nature 424, 810 (2003).

22. Bergmann, K., Theuer, H. \& Shore, B. W. Coherent population transfer among quantum states of atoms and molecules. Rev. Mod. Phys. 70, 1003 (1998)

\section{Acknowledgements}

M.M. thanks C. Fort and G. Modugno for useful discussions and suggestions. This material is based upon work supported by NSFC (Grant Nos. 11375025, 11075013, 11005099), Fundamental Research Funds for the Central Universities (Grant No. 201013037), an Ikerbasque Foundation Startup, the Basque Government (grant IT472-10), the Spanish MICINN (Project No. FIS2012-36673-C03-03), the UPV/EHU under program UFI 11/55, the Research Grant Council of Hong Kong (Grant No. HKU8/CRF/11G) and the Croucher Foundation under the Croucher Innovation Award.

\section{Author contributions}

Z.-M.W. contributed to numerical analysis of theoretical models and prepared the first version of the manuscript, L.-A.W. to the conception and design of this work, and M.M. to the experimental proposal. All authors (Z.-M.W., L.-A.W., M.M., W.Y. and B.S.) participated in the discussions and the writing of the manuscript.

\section{Additional information}

Competing financial interests: The authors declare no competing financial interests.

How to cite this article: Wang, Z.-M., Wu, L.-A., Modugno, M., Yao, W. \& Shao, B. Fault-tolerant almost exact state transmission. Sci. Rep. 3, 3128; DOI:10.1038/srep03128 (2013).

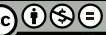

This work is licensed under a Creative Commons AttributionNonCommercial-NoDerivs 3.0 Unported license. To view a copy of this license, visit http://creativecommons.org/licenses/by-nc-nd/3.0 